5. Medeiros FA, Zangwill LM, Bowd C, et al. Use of progressive glaucomatous optic disk change as the reference standard for evaluation of diagnostic tests in glaucoma. Am J Ophthalmol 2005;139:1010-1018.

\section{Prevention of Dermatologic Side Effects of Bimatoprost $0.03 \%$ Topical Therapy}

\author{
Marco Centofanti, MD, $\mathrm{PhD}$, \\ Francesco Oddone, MD, Sergio Chimenti, MD, \\ Lucia Tanga, MD, Luigi Citarella, MD, \\ and Gianluca Manni, MD
}

PURPOSE: To investigate the efficacy of reducing the drop-skin contact to prevent dermatologic side effects of bimatoprost $0.03 \%$ topical therapy.

DESIGN: Prospective, randomized, single-blinded, internally controlled study.

METHODS: Enrolled subjects started bimatoprost $0.03 \%$ therapy once at night in both eyes and were instructed to wipe selectively only one eye (eye 1) with an adsorbent pad during and after drops administration for four months. The fellow eye acted as the internal control. Eyelash growth, regional skin hypertrichosis, and pigmentation on the periocular skin were assessed at baseline and during the four months of follow-up. RESULTS: A lower incidence of eyelash growth and skin pigmentation in the inferonasal pericanthal region were observed in eye 1 . The incidence of pigmentation in the inferotemporal skin region and skin hypertrichosis were similar in the two eyes.

CONCLUSION: The reduction of the drop-skin contact affects the regional incidence and the extent of dermatologic skin changes that are related to bimatoprost 0.03\% topical therapy. (Am J Ophthalmol 2006; 142:1059-1060. (C) 2006 by Elsevier Inc. All rights reserved.)

P ROSTAGLANDINS AND PROSTAMIDES ANALOGS ARE widely recognized to be the most effective intraocular pressure-lowering agents; however, despite the systemic safety, the clinical use of these drugs is often limited by the occurrence of dermatologic side effects, such as changes of length and density of eyelashes, ${ }^{1}$ changes of length and density of vellus hairs of the malar and external canthal

\section{Accepted for publication Jul 4, 2006}

From the Departments of Ophthalmology (M.C., L.T., G.M.) and Dermatology (S.C., L.C.), University of Tor Vergata, Rome, Italy Fondazione GB Bietti for Study and Research in Ophthalmology, IRCCS, Rome, Italy (F.O.).

Inquiries to Francesco Oddone, MD, Largo Olgiata 15, isola 75, 5-4, 00123, Rome, Italy; e-mail: f.oddone1@virgilio.it skin regions, ${ }^{2}$ and changes in periocular skin pigmentation. ${ }^{3}$ The occurrence of these changes is likely to be related to the drop-skin contact; the purpose of this study was to investigate the efficacy of reducing the drop-skin contact to prevent topical dermatologic side effects of bimatoprost $0.03 \%$ topical therapy.

This prospective, parallel, randomized, single-blinded, internally controlled clinical study was approved by the Institutional Ethics Committee, and each subject signed a written informed consent form. Inclusion criteria were the diagnosis of glaucoma or ocular hypertension in both eyes. Exclusion criteria were the past or current use of topical prostaglandins or prostamides, the need for more than one drug to control intraocular pressure, concomitant active ocular or dermatologic diseases, the use of sunless tanning products, or tanning booths in the past six months.

After washout, subjects started bimatoprost $0.03 \%$ topical therapy once at night in both eyes and were trained to blot adequately only one randomly selected eye (eye 1 ) with a provided disposable adsorbent pad (TNT $7.5 \times 7.5$ $\mathrm{cm}, 4$ layers; Ploing SRL Merchandising, Pomezia, Rome, Italy) every day during and after drop administration. Eye 2 acted as an internal control (control). Eyelash length was quantified (at baseline and at months 1, 3, and 4) by the removal and measurement under a microscope of the longest eyelash of the upper eyelid of both eyes. ${ }^{1}$ Skin hypertrichosis and pigmentation were assessed by three investigators (who were blinded to the data) by means of digital contact dermatoscopy ${ }^{4}$ (Dermaphot; Heine Optotechnik, Herrsching, Germany). The study took place during the winter, and the patients were asked not to use any make-up products the visit days and any sunless tanning product or tanning booths throughout the study period.

An electronic web-based randomization plan generator was used for randomization. One-way analysis of variance test, paired $t$ test, $\chi^{2}$ test, and Fisher exact test were used to analyze the data. Eyelash growth was defined as a change of eyelash length of $>1.5 \mathrm{~mm}$ from baseline. Twenty eyes from 10 patients were included in the statistical analysis (seven women, three men; mean age, $56.2 \pm 16.6$ years; range, 31 to 79 years). Baseline variables were similar between eyes. A trend of growth was observed in both eyes during the study (Table), although changes from baseline were significantly greater in the control eye at month 3 and at month 4 (Figure 1).

The incidence of eyelash growth (number of eyes with induced eyelash growth/number of eyes examined) was higher in the control eye at month 4 compared with eye 1 (Figure 2). A lower incidence of skin hyperpigmentation from baseline was observed in the inferonasal pericanthal skin region in eye 1 , compared with the control eye at month $4(10 \%$ vs $60 \% ; P=.029)$. The incidence of hyperpigmentation in the inferotemporal pericanthal skin 
TABLE. Eyelash Length (mean \pm SD and \% of change from baseline) Measured During Bimatoprost $0.03 \%$ Topical Therapy

\begin{tabular}{cccccc}
\hline & Baseline & Month 1 & Month 3 & Month 4 & $P$ \\
\hline Eye $1^{*}(\mathrm{~mm})$ & $8.5 \pm 1.0$ & $8.7 \pm 1.7$ & $10.0 \pm 1.9$ & $10.3 \pm 1.9$ & 0.001 \\
Control $^{\dagger}(\mathrm{mm})$ & - & $(+2.3 \%)$ & $(+18.8 \%)$ & $1.21 .3 \%)$ & 0.002 \\
& $8.8 \pm 1.5$ & $9.5 \pm 1.7$ & $(+29.7 \%)$ & $(+36.8 \%)$ \\
\hline
\end{tabular}

$\mathrm{SD}=$ standard deviation

*Intervention eye, where the drop-skin contact has been minimized during drop administration throughout the study.

†Fellow eye (internal control) where no special care has been taken to prevent the drop-skin contact. Although length changes from baseline were greater in the control eye, a statistically significant trend of growth was observed in both eyes.

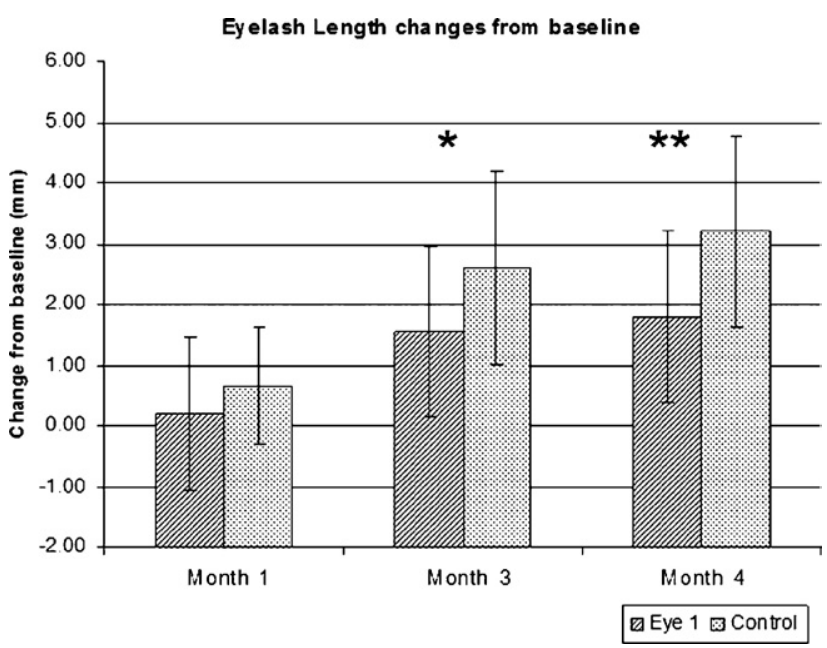

FIGURE 1. Eyelash length changes from baseline at each time point during bimatoprost $0.03 \%$ topical therapy. Length changes have been found to be smaller in the eye in which the drop-skin contact had been minimized (eye 1$)$ both at month $3(* P=.021)$ and at month 4 $(* * P=.003)$ compared with the control eye (control) with which no special care had been taken to prevent the dropskin contact.

(40\% vs $60 \% ; P=.32$ ) and the incidence of skin hypertrichosis $(60 \%$ vs $60 \%$; $P=.67)$ were similar between eye 1 and the control eye.

Dermatologic changes that are related to prostaglandins analog topical therapy are likely to be related to an alteration of the transition between phases of the hair cycle, ${ }^{5}$ and the periocular drop-skin contact is believed to be necessary to promote these changes. The results of this study showed that the minimization of the drop-skin contact during drop administration allows the reduction of eyelash growth and skin hyperpigmentation in the inferonasal periocular skin region, but not in the inferotemporal region. This regional difference may be related to a lower reliability of the study intervention to prevent the drop-skin contact in the temporal region. The

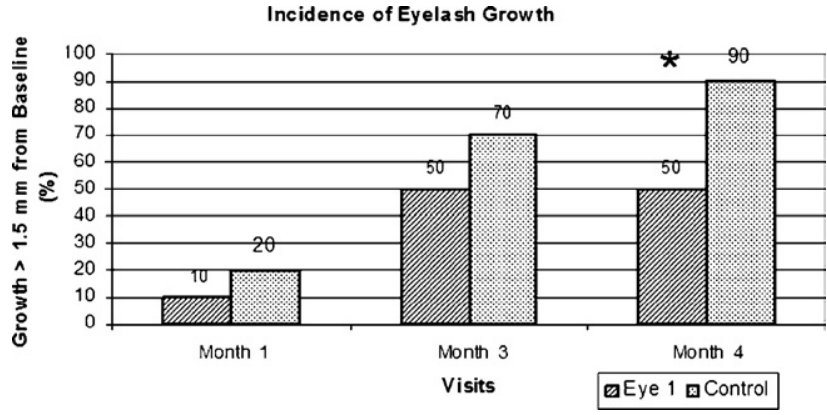

FIGURE 2. Incidence of eyelash growth defined as a length change $>1.5 \mathrm{~mm}$ from baseline. After four months of bimatoprost $0.03 \%$ topical therapy, a statistically lower incidence of eyelash growth $(* P=.011 \%)$ was observed in the eye in which the drop-skin contact had been minimized (eye 1) compared with the control eye (control) with which no special care had been taken to prevent the drop-skin contact.

finding of a positive effect of the intervention may stimulate further studies about possible ways for a reliable prevention of the drop-skin contact in the whole periocular area.

\section{REFERENCES}

1. Sugimoto M, Uji Y. Quantitative analysis of eyelash lengthening following topical latanoprost therapy. Can J Ophthalmol 2002;37:342-345.

2. Hart J, Shafranov G. Hypertrichosis of vellus hairs of the malar region after unilateral treatment with bimatoprost. Am J Ophthalmol 2004;137:756-757.

3. Wand M, Ritch R, Isbey EK Jr, Zimmerman TJ. Latanoprost and periocular skin color changes. Arch Ophthalmol 2001; 119:614-615.

4. Carli P, de Giorgi V, Salvini C, Mannone F, Chiarugi A. The gold standard for photographing pigmented skin lesions for diagnostic purposes: contact vs distant imaging. Skin Res Technol 2002;8:255-259.

5. Johnstone MA, Albert DM. Prostaglandin-induced hair growth. Surv Ophthalmol 2002;47:S185-S202. 